(C) 2015 IEEE. Personal use of this material is permitted. Permission from IEEE must be obtained for all other uses, in any current or future media, including reprinting/republishing this material for advertising or promotional purposes, creating new collective works, for resale or redistribution to servers or lists, or reuse of any copyrighted component of this work in other works. 


\title{
Transradial Amputee Gesture Classification using an Optimal Number of sEMG Sensors: An Approach using ICA Clustering
}

\author{
Ganesh R. Naik, Senior Member, IEEE, Ali Al-Timemy, Member, IEEE, and Hung T. Nguyen, \\ Senior Member, IEEE
}

\begin{abstract}
Surface electromyography (sEMG) based pattern recognition studies have been widely used to improve the classification accuracy of upper limb gestures. Information extracted from multiple sensors of the sEMG recording sites can be used as inputs to control powered upper limb prostheses. However, usage of multiple EMG sensors on the prosthetic hand is not practical and makes it difficult for amputees due to electrode shift/movement, and often amputees feel discomfort in wearing SEMG sensor array. Instead, using fewer numbers of sensors would greatly improve the controllability of prosthetic devices and it would add dexterity and flexibility in their operation. In this paper, we propose a novel myoelectric control technique for identification of various gestures using the minimum number of sensors based on Independent Component Analysis (ICA) and Icasso clustering. The proposed method is a model based approach where a combination of source separation and Icasso clustering was utilized to improve the classification performance of independent finger movements for transradial amputee subjects. Two sEMG sensor combinations were investigated based on the muscle morphology and Icasso clustering and compared to Sequential Forward Selection (SFS) and greedy search algorithm. The performance of the proposed method has been validated with 5 transradial amputees, which reports a higher classification accuracy $(>95 \%)$. The outcome of this study encourages possible extension of the proposed approach to real time prosthetic applications.
\end{abstract}

Index Terms-Blind source separation (BSS); Independent component analysis (ICA); Finger Gestures; Linear discriminant analysis (LDA); Myoelectric control; Pattern Recognition, Surface electromyography (sEMG); Transradial Amputees.

\section{INTRODUCTION}

$\mathrm{I}_{\mathrm{e}}^{\mathrm{N}}$ Nrecent years, myoelectric control based on surface electromyography (sEMG) has been the focus of the research on myoelectric prosthesis (MP) technology [1-4].

Manuscript received January, 29, 2015, "This work was supported by the UTS Chancellor's Postdoctoral Research Fellowship Grant".

Ganesh R. Naik and Hung T Nguyen are with Centre for Health Technologies (CHT), University of Technology Sydney, 2007, Australia (email: Ganesh.Naik@uts.edu.au; Hung.Nguyen@uts.edu.au).

Ali Al-Timemy is with Biomedical Engineering Department, Al-Khwarzmi College of Engineering, Baghdad University, Iraq and Centre for Robotics and Neural Systems (CRNS), Cognition Institute, School of Computing and Mathematics, Plymouth University, United Kingdom (UK) (e-mail: ali.altimemy@kecbu.uobaghdad.edu.iq, ali.ali@plymouth.ac.uk)
Currently, most of the EMG-controlled device users are transradial upper-limb amputees (amputation occurred below elbow). For these people, the replacement of missing arm functionalities can be of a significant improvement to the quality of life [5-7]. Surface EMG based pattern recognition (PR) is achieved by applying pattern recognition algorithm to EMG signal stream. The approach consists of four sequential steps: (i) EMG signal conditioning and pre-processing, (ii) EMG feature extraction, (iii) EMG dimensionality reduction and (iv) EMG pattern classification [8].

Surface EMG pattern recognition techniques have been developed to increase dexterity of myoelectric control and to overcome the limitations of conventional proportional control by extracting multiple features from EMG signals rather than entirely relying on EMG amplitude [9]. In order to control a sEMG based prosthetic hand, it is essential to map EMG signals corresponding to different muscle contractions using various pattern recognition and classification methods[6]. However, in most of the instances, the above is achieved either by usage of larger number of EMG sensors or through EMG sensor arrays. Despite the encouraging results and higher classification accuracies from high-density sEMG recording and analysis, using a large number of EMG sensors hinders the practical application in terms of computational complexity and practicality. Therefore, for myoelectric prosthesis, it is very important to select a small number of appropriate sensors which can yield the desired classification accuracy. Moreover, it is essential to understand which muscles are relevant and how they are connected to each hand or finger movement. There exist numerous barriers which limit clinical implementation of PR systems. Among these are impractically large number of EMG sensors [10], variations in limb position $[3,11]$ and force variation $[12,13]$. Usage of large number of EMG sensors may cause patient discomfort, in addition to the computational and hardware costs [14]. To improve the practicality and usability of PR systems, finding the optimal set of EMG sensors is an important challenging task. The benefits of reduced subset of EMG sensors can be seen not only in the laboratory controlled experiments, but also in the real time clinical prostheses usage which will have an important role to enable the clinical implementation of PR systems $[4,15]$.

In ideal situations, prosthetic devices use large number of 
electrodes for gesture recognition. However, it is difficult to extract the reliable information (sources) and reduce the number of sensors (at the same time) due to the amputation level and muscle overlapping. In recent times, several research studies are carried out to tackle the above issue. A collection of sEMG sensor minimisation techniques are concisely presented below to emphasise the suitability of source separation technique for the task at hand. In order to reduce the number of sensors of myoelectric classification, previously, researchers have used a sequential forward selection (SFS) method [16]. The method selects the best single sensor for classification and then adds one sensor at a time that can maximize classification performance in combination with the selected sensors. With this method, the classifier has to be repeatedly implemented in order to select a single sensor each time. Moreover, reduction of the number of features in the same sensor is not considered, since features extracted from each sensor are fixed during the process. Thus, the SFS algorithm cannot remove the already selected features that may become obsolete after the addition of other candidate features (or sensors). In order to overcome the limitations of SFS, Liu et al [9], proposed a sensor reduction strategy based on evaluation of EMG features extracted from high-density surface EMG recordings. The proposed strategy showed an improvement and does not require repeated implementation of the classification, but such a system may not be practical for prosthetic devices due to the use of high density sensor arrays. Recently, Geng et al. [17] proposed a multi-class common spatial pattern (MCCSP) for EMG sensor selection for high density EMG recorded from impaired traumatic brain injury patients. The proposed method outperformed the traditional SFS and Fisher-Markov selector (FMS) in terms of finding the optimal EMG features for gesture classification. While the above studies showed significant improvement in reduction of sEMG sensors, identifying the best choice of sensors still remains an important challenge for EMG prosthetics. Also, since the sEMG signals are estimated from the front and back side of the forearm muscles [18, 19], a combination of source separation and careful selection of muscle configuration would help to minimise the number of sEMG sensors.

Independent components analysis (ICA) is one of the blind source separations (BSS) techniques which utilises both lower and higher order statistics to estimate set of linearly mixed variables into their independent components (ICs). In the recent past, ICA has been extensively used for sEMG signal processing, especially for upper limb EMG applications [2022]. One of the advantages of using ICA is that it decomposes the linearly mixed several muscle activities into its constituent ICs or motor units [20, 22].

Extracting most reliable information from the ICA algorithms are always a challenging task, because they (most algorithms) have random (stochastic) elements [23]. The most reliable ICs provide important neural information for the myoelectric classification. One solution in this regard is to use Icasso, an extension of ICA, which pools all the IC estimates together and forms clusters bottom-up among them. The basic idea is that a tight cluster of estimates is considered to be a candidate for including a "good" estimate. A centroid of such cluster is considered more reliable estimates than any estimate from an arbitrary run[24]. In the recent past, Icasso has been successfully used for various biomedical signal processing applications such as artefact removal [25], measuring directional coupling between electroencephalography (EEG) sources [26], brain functional connectivity [27], and repeatability measures on results of fMRI [28]. To the best of our knowledge, this study reports the first use of Icasso clustering for EMG signal analysis for prosthesis control applications. In this research, Icasso is being used to find the underlying sEMG sources and to find a subset of useful electrodes contributing to those sources.

The main aim of this study is to propose a novel myoelectric classification scheme using minimum number of EMG sensors. In order to minimise the number of EMG sensors, two strategies are used in this study: i) usage of Icasso [24] to cluster the number of similar muscle activities (sensors) as groups and ii) maximum number of times the sensors appeared in the cluster. The features extracted from the reduced sEMG sensors are then classified using linear discriminant analysis (LDA) to identify movements associated with the different finger gestures for five transradial amputees.

A brief overview of the ICA, followed by a succinct description of the Icasso is in order in Section II. The experimental design, feature extraction methods and LDA classifier design for the proposed scheme are presented in Section III. Subsequently, the experimental results that corroborate with our claim are provided in Section IV. Finally, Section V includes conclusions drawn and future challenges associated with myoelectric classification and prosthesis.

\section{BACKGROUND}

\section{A. Independent Component Analysis}

ICA is one of the blind source separation (BSS) techniques and its goal is to separate/estimate instantaneously mixed sources from the recordings. Here, we briefly recall the main concept associated with the ICA. Let $M$ be the number of sEMG amputee surface measurements $x_{i}(t), i \in[1, M]$ to which contribute $\mathrm{N}$ unknown sEMG sources $s_{i}(t), i \in[1, N]$, with $N \leq M$. The aim of ICA is to find the unknown muscle components (s) into their motor unit action potential trains MUAPS/ICs, such that $\mathrm{x}=\mathrm{As}$, where $\mathrm{A}$ is called the mixing matrix (without any prior information)[20,29]. ICA computes the unmixing matrix $\mathrm{W}$ and estimate the sEMG sources $s$ provided $(\mathrm{s}=\mathrm{Wx})$; they are statistically independent from each other[23, 30]. ICA can be solved using both higher order and lower order statistical techniques such as FastICA [23], Infomax [30], JADE and SOBI. This study uses FastICA algorithm, which adapts a fixed point iteration scheme to compute the maximum of the non-Gaussianity of the sources. More details of FastICA can be found in [23].

\section{B. Icasso and Optimum Sensor Selection}

The FastICA algorithm is stochastic in nature, which means that multiple repetitions of the ICA analysis on the same 
dataset can give slightly different results. One of the solutions is clustering of the obtained components based on multiple ICA runs, which is also known as Icasso [24]. The method is based on estimating a large number of independent components, and visualizing their clustering in the signal space. Each estimated IC is one point in the space. If an IC is reliable, (almost) every run of the algorithm should produce a point that is very close to the ideal component corresponding to the cluster center. Thus, reliable independent components correspond to tight clusters, and unreliable ones correspond to points which do not belong to any cluster. Icasso identify clusters of ICA-estimates that are consistently found across random initializations of the ICA algorithm of the input data. The process of Icasso consists of the following steps [24]:

1) Initially, parameters (symmetrical or deflatory, contrast function, etc.) for the estimation of FastICA algorithm are selected.

2) The estimation is run several times using the selected training parameters. Each time the data is bootstrapped and/or the initial conditions of the estimation algorithm are changed.

3) Mutual similarities between all the estimates are computed. As the measure of similarity, we use the absolute value of the linear correlation coefficient between the ICs. The estimates are clustered (agglomerative clustering with average-linkage criterion) according to their mutual (dis) similarities.

4) The clustering is visualized as a dendrogram and a $2 \mathrm{D}$ plot. The user investigates how dense the clusters are. The clustering of the estimates is expected to yield information on the reliability (robustness) of estimation. A compact cluster emerges when a similar estimate repeatedly comes up despite of the randomization.

5) The user can retrieve the estimates belonging to certain cluster(s) for further analysis and visualization.

The mathematical basis of Icasso algorithm is explained in detail in [24]. However, the cluster combination for each gesture may slightly vary; this is due to electrode shift or level of Transradial amputation. Hence, Icasso algorithm was utilised to select the best four EMG sensors responsible for amputee finger gestures. This would also help us to determine functional synergy of the muscles.

\section{METHODOLOGY}

\section{A. Amputee Participants}

The EMG dataset used in this study was originally recorded by Al-Timemy et al. [7]. The EMG signals were acquired from the left stump of five traumatic transradial amputees (TR1-TR5) aged 24-34 years. It is noteworthy to mention that none of the amputees used myoelectric prosthesis after the amputation. The amputees' data were collected at the Artificial Limbs and Rehabilitation Centres in Baghdad (Iraqi Army) and Babylon (Ministry of Health), Iraq. It may be noted that the time since amputation was within the range of 4-8 years for all amputees. The study was approved by the Human Ethics Committee of the Faculty of Science and Technology at Plymouth University (17/9/2009) and updated to collect the data from the Iraq. Amputee subjects were

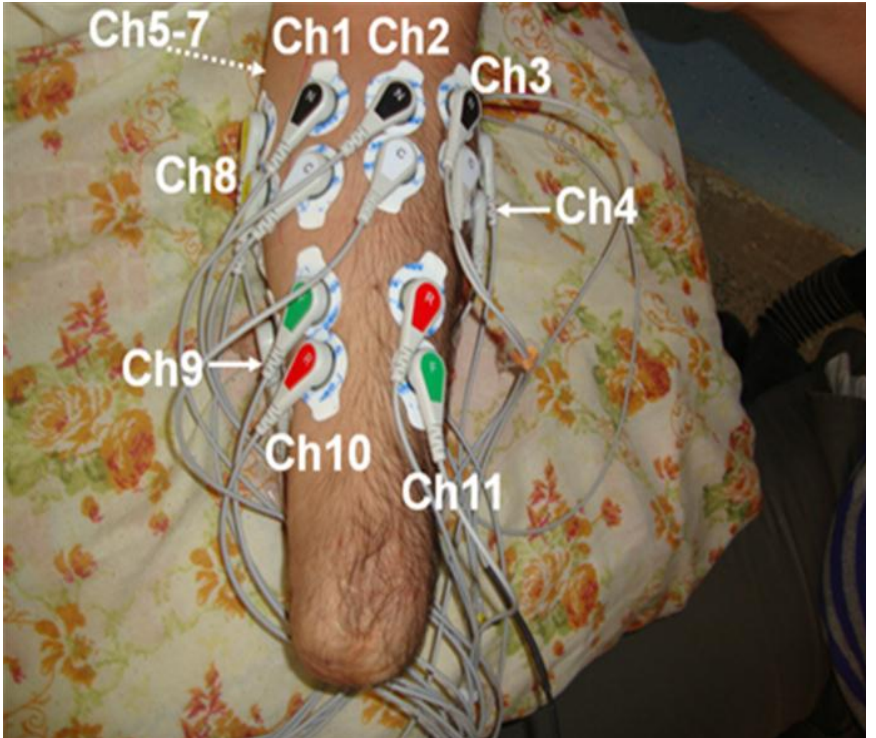

Fig. 1. Example of electrode locations for amputee person TR5

debriefed about the study, and they were asked to give their written informed consent to participate in the study.

\section{B. Electrode Placement}

Before the start of the EMG data collection, the skin was cleaned with alcohol and abrasive skin preparation gel (NuPrep ${ }^{\circledR}$, D.O. Waver and Company, USA) was applied to the stump. Eleven EMG sensors connected to a differential amplifier were used with pairs of self-adhesive $\mathrm{Ag}-\mathrm{AgCl}$ electrodes (Tyco healthcare, Germany) placed around the circumference of the upper part of the forearm either in one or two rows. To reproduce electrode positions, European recommendations for sEMG (SENIAM) [31] were followed. The elbow joint was used as a reference to mark the electrode locations on the upper part of the forearm. For TR1, the 11 pairs of sensors were placed around the circumference of the upper forearm in one row, whereas the sensors were placed into two rows around the circumference of the upper forearm for the rest of the amputees. Fig.1 illustrates an example of the locations of EMG sensors for TR5. The ground reference electrode was placed at the end of the stump for TR1-TR2 and at the Olecranon process of the Ulna for TR3-TR5. Bipolar EMG measurements were used with inter-electrode distance of $24 \mathrm{~mm}$ as recommended by Young et al. and the SENIAM [20]. It is worth mentioning that the amputees TR1, TR3 and TR4 have thick hair on their forearms but it was not shaved to avoid inconvenience to the amputees.

\section{Experimental Procedure}

The EMG signals were acquired with the custom-built EMG amplifier. Each EMG sensor was sampled at a rate of $2000 \mathrm{~Hz}$ with data acquisition system (NI USB 6210, National Instruments, USA) with 16-bit resolution. A Virtual Instrument (VI) was developed in LABVIEW (National Instruments, USA) to display and store the EMG signals.

None of amputee subjects had been trained on EMG recording prior to the study. The amputees were instructed to produce a specific imagined finger movement. They used the 


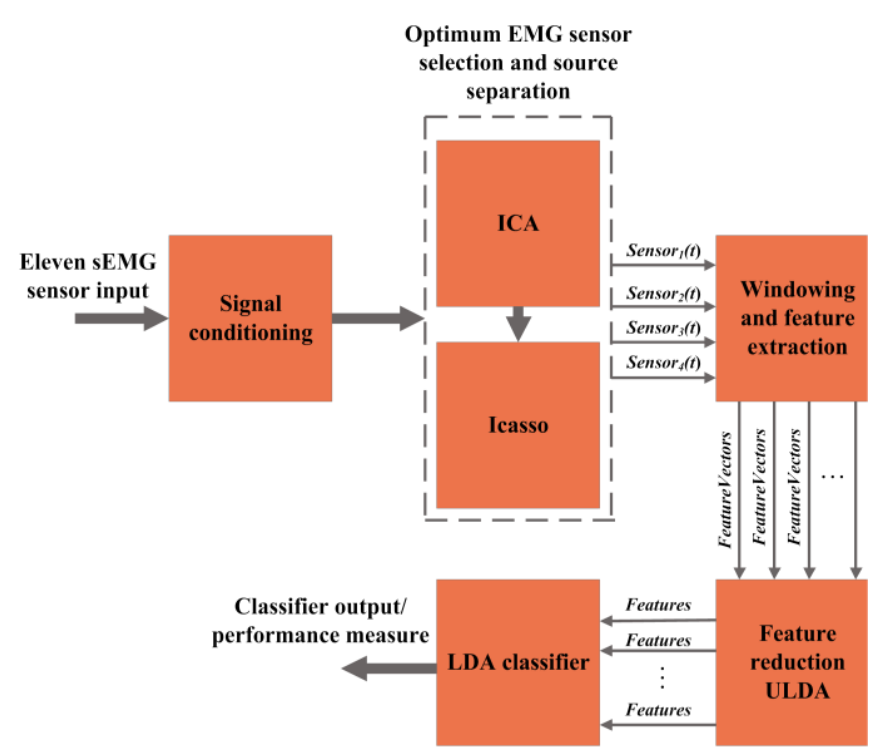

Fig. 2. Schematic diagram of the Icasso-based amputee gesture classification method proposed in this study.

movements of the fingers of the intact-hand to help them to reproduce the desired movement. They performed 12 classes of finger movements (11 individual finger movements as well as the rest position, which is considered as one of the movement classes in this study). The 12 individual finger movements performed by amputee subjects are: little flexion (f1), ring flexion (f2), middle flexion (f3), index flexion (f4), rest position, little extension (e1), ring extension (e2), middle extension (e3), index extension (e4), thumb flexion (f5), thumb extension (e5) and thumb abduction (a5). Thumb adduction was discarded in the data recording, because the muscles responsible for thumb adduction lie in the hand itself and it cannot be decoded from the upper forearm.

During the recording of the EMG signals, each participant sat on a chair in front of a computer with the Labview interface screen to view all the EMG sensors in real-time, while imagining the movements. The arm was resting on a pillow, and participants were instructed to keep their arm position fixed during the experiment. They were asked to produce a succession of different finger movements separated by 5-10 second periods of rest. Participants were asked to produce finger movements with a moderate, constant-force, and non-fatiguing contraction to the best of their ability. The final position of a movement was held for a period of 5-10 seconds for the amputees in order to avoid fatigue. Each holding phase is referred to in this study as a "trial". TR1 and TR2 performed shorter trials of 3-4 seconds than the rest of the amputees. The transition regions were removed from the signals. Five-seven trials were recorded for each movement of the 11 movements for each amputee.

\section{Data processing - Feature Extraction and Feature reduction}

Prior to feature extraction, movement artefact $(<20 \mathrm{~Hz})$, power-line interference $(50 \mathrm{~Hz})$ and high-frequency noise $(>450 \mathrm{~Hz})$ were removed. The sEMG data were divided into overlapping windows of $256 \mathrm{~ms}$ length with a $64 \mathrm{~ms}(25 \%)$ increment between windows. This segmentation scheme was used for all numerical experiments in this study. The proposed amputee gesture classification scheme based on ICA and Icasso clustering is shown in Fig. 2.

In order to select the optimum sensor configuration, we used Icasso's default agglomerative clustering with averagelinkage criterion. Icasso runs the ICA algorithm (FASTICA algorithm was used) a number of times (20 runs were used); each run gives different estimates of the ICs. Icasso collects the ICs estimated from all runs and then matches components across runs by clustering components based on the absolute value of the correlation between squared source estimates. The number of clusters in the data was automatically selected with the R-index[24]. Each cluster was uniquely represented by a single centrotype ICA-estimate, which is just an estimate in the cluster that has the maximum sum of similarities to other points in the cluster. Only centrotypes of significant clusters were considered as valid ICA-estimates.

Fig. 3(a) and Fig. 3(b) depict the results of the Icasso analysis applied to sEMG recordings from amputee TR1 and TR2 respectively where Dendrogram is a tree-structured graph used to visualize the result of a hierarchical clustering. The Rindex suggested an optimal partition of 4 clusters in the data. This index measures the difference between average intracluster correlations and average intercluster correlations for each cluster, which is useful in quantifying the consistency in the performance of the algorithm and also selection of optimal partition of data [24]. These four clusters exhibited a high repeatability within the same subject and across subjects. For instance, Fig. 3 (a) shows sensors $(5,10,3)$, (9, 8, 6), (7, $2,4)$ and $(1,11)$ as four clusters for amputee 1 (TR1). Similar configurations are achieved for the other amputees as well. The Icasso cluster combinations obtained for five amputees are shown in Table I. From the table, it can be seen that sensors 3, 9, 7 appear in all cluster combinations (amputees) and hence, they (3 sensors) were selected for further analysis. Whereas, sensors 1 and 2 are considered for 4th sensor combination as they appear at 4 and 3 occasions respectively. The final sensor combinations consisted of sensors 2,3,7,9 and sensors $1,3,7,9$ which were considered for further analysis. The choice of optimal sensors (final 4 sensors) required for amputee gestures were later decided based on classification accuracy obtained using these sensor combinations.

To extract useful information from the segmented sEMG signals, 9 time domain features were extracted for each EMG sensor which includes a fourth-order auto regression (AR) model, root mean square (RMS), mean absolute value (MAV), variance (VAR), waveform length (WL) and zero crossing (ZC). These features were chosen based on the fact that time domain features such as AR and RMS can achieve higher performance than that of other feature extraction methods such as Fourier transform and wavelet transform for the detection of various hand and finger movements with EMG signals [7, 17, 20]. Furthermore, it has been shown that AR coefficients are computationally efficient and robust to displacements in electrode positions $[7,32-35]$. 
TABLE I

OVERALL CLUSTER COMBINATIONS FOR 11 SEMG SENSORS USING ICASSO FOR 5 TRANSRADIAL AMPUTEES

\begin{tabular}{|c|c|c|c|c|}
\hline $\begin{array}{c}\text { Amputee } \\
\text { ID }\end{array}$ & Cluster 1 & Cluster 2 & Cluster 3 & Cluster 4 \\
\hline TR1 & $5,10,3$ & $9,8,6$ & $7,2,4$ & 1,11 \\
\hline TR2 & $11,3,2$ & 9,5 & $7,8,6$ & $1,10,4$ \\
\hline TR3 & $3,8,5$ & 9,4 & $7,11,10$ & $1,2,6$ \\
\hline TR4 & 3,6 & $11,9,10$ & $7,1,4$ & $2,8,5$ \\
\hline TR5 & $11,3,4$ & $9,10,2$ & $5,7,8$ & $1,6,2$ \\
\hline
\end{tabular}

Feature reduction: In order to reduce the dimensionality and to improve the class separability of extracted features, uncorrelated linear discriminant analysis (ULDA) feature projection technique was used. ULDA is a supervised method which uses features with class labels and maximises the ratio of the between-class distance to the within-class distance[36]. However, this method suffers from the problem of singularity in the scatter matrix that occurs when the feature vector dimension is much larger than the sample size [37] which is not applied to our EMG data since our sample size is much larger than the feature vector dimension. ULDA is an enhancement to LDA, which imposes the additional requirement that reduced features be statistically uncorrelated with one another; hence, minimising redundancies. It will reduce the size of the features to $c-1$ feature where $c$ is the number of classes ( $c=11$ for the current study).

\section{E. Data Classification and Cross-Validation using LDA}

In order to ascertain the robustness of the proposed gesture recognition scheme, the choice of classifier and statistical methods are very important. The previous studies reported in literature have used numerous classification schemes for EMG data, most popular among them include SVM [6], Neural network, k-Nearest Neighbors (kNN) and LDA [6]. For the amputee gesture data, classification is simply performed using LDA classifier. The advantage of using LDA is that it does not necessitate iterative training, which avoids the potential for under or over-training of the data [38]. Furthermore, using LDA, a high dimensionality problem can be well linearized during feature reduction. For each gesture, the first 3 trials were combined in one file to produce the training set while the rest of the trials were used as testing set. The training and testing sets were kept separately to calculate the classification error. To quantify the performance of the proposed method, the overall classification error (CE) was computed using:

$$
C E=\frac{\text { Number of misclassified testing data }}{\text { total number of applied testing data }} \times 100 \%
$$

In addition, confusion matrix (CM) which calculates the test results between predicted classes and the actual classes was also computed.

To test the statistical significance between the 2 EMG sensor combinations, an independent-samples Mann-Whitney (nonparametric $t$ test) test was applied to find differences in classification errors of the two sensor combination schemes $(1,3,7,9$ and $2,3,7,9),(1,3,7,9$ and SFS), and $(1,3,7,9$ and Greedy search algorithm), respectively. This test assumes that results of the two distributions are identical.

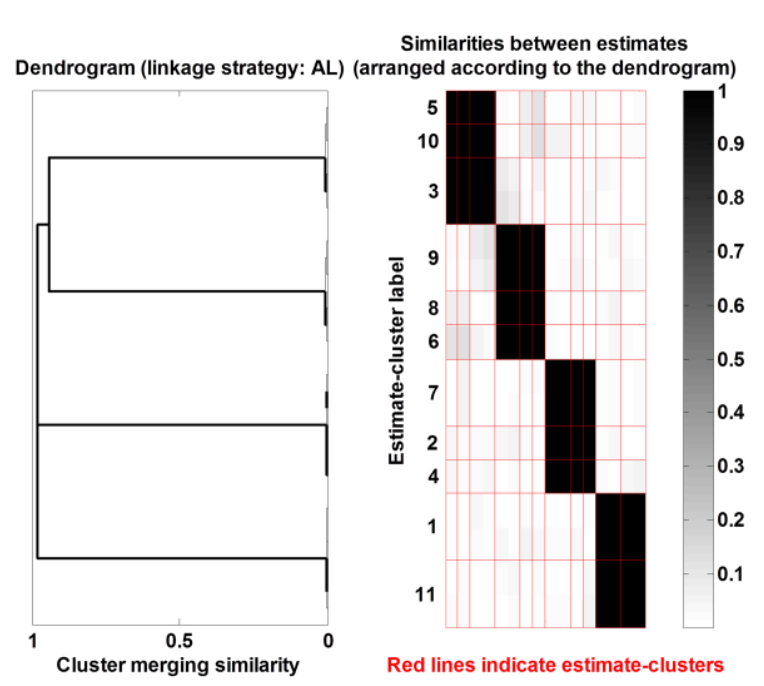

Fig. 3(a)

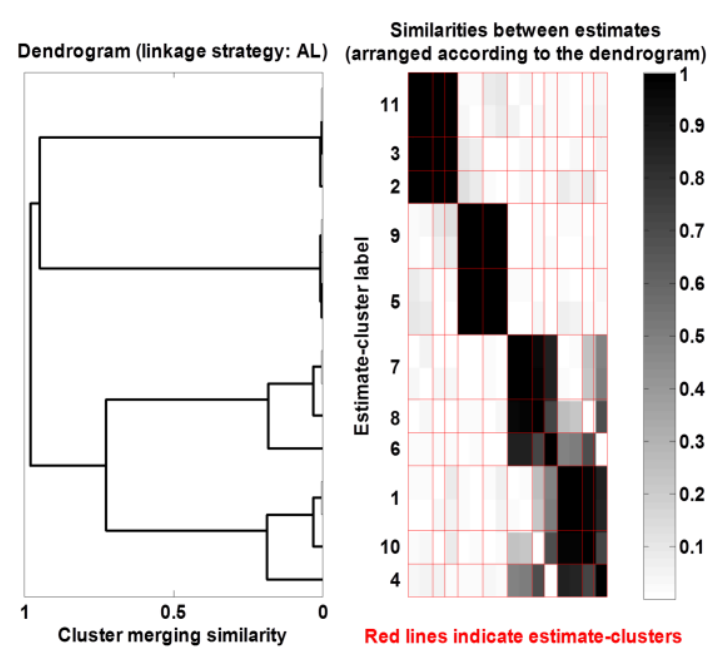

Fig. 3(b)

Fig. 3. (Left panel) Dendrogram (a tree-structured graph used to visualize the result of a hierarchical clustering) illustrating the arrangement in 11 sEMG sensors (as suggested by the R-index) of the ICA-estimates obtained with Icasso (a) for TR1 (b) for TR2. In both figures, the horizontal axis signifies the dissimilarity values at which clusters are merged at each possible partition level. (Right panel) The vertical axis indexes ICA-estimates as 4 major clusters. The colour scale indicates the cross-correlation coefficient between the muscle configurations of individual ICA-estimates. Clusters of ICA-estimates are indicated with red lines and their corresponding labels are depicted in the left vertical axis.

\section{F. Effect of the Number of Sensors on Classification Performance}

In order to measure the efficacy of the proposed ICA clustering based sensor selection algorithm it is compared with two other popular methods 1) SFS [16, 17] and 2) sensor elimination technique (greedy search algorithm) [7]. The two methods are briefly explained below. The SFS is an iterative searching technique, in which one optimal sensor that produces the highest classification accuracy was firstly selected among all the sensors, and then another sensor that can achieve the maximum classification accuracy with the combination of the selected sensors are added. On the other hand, in a sensor elimination technique the main objective is 
TABLE II

AVERAGE CLASSIFICATION ACCURACY FOR FIVE TRANSRADIAL AMPUTEE SUBJECTS USING ALL SENSORS, 2 DIFFERENT SENSOR COMBINATIONS $(1,3,7,9)$ AND $(2,3,7,9)$, SFS AND GREEDY SEARCH ALGORITHM.

\begin{tabular}{|c|c|c|c|c|c|}
\hline Classes & $\begin{array}{c}\text { Classification Accuracy } \\
\text { (Mean } \pm \text { SD)Sensors } \\
(1,3,7,9)\end{array}$ & $\begin{array}{c}\text { Classification Accuracy } \\
\text { (Mean } \pm \text { SD)Sensors } \\
(2,3,7,9)\end{array}$ & $\begin{array}{c}\text { Classification Accuracy } \\
(\text { Mean } \pm \text { SD }) \\
\text { All sensors - using ICA }\end{array}$ & $\begin{array}{c}\text { Classification Accuracy } \\
\text { (Mean } \pm \text { SD) } \\
\text { All sensors - SFS }\end{array}$ & $\begin{array}{c}\text { Classification Accuracy } \\
\text { (Mean } \pm \text { SD) } \\
\text { All sensors -Greedy } \\
\text { search method } \\
\end{array}$ \\
\hline Little flexion (f1) & $98.6 \pm 1.4$ & $91.5 \pm 1.2$ & $95.4 \pm 1.5$ & $92.4 \pm 0.8$ & $91.2 \pm 1.1$ \\
\hline Ring flexion (f2) & $97.8 \pm 1.2$ & $90.2 \pm 1.4$ & $93.2 \pm 1.1$ & $91.4 \pm 1.2$ & $90.2 \pm 0.7$ \\
\hline Middle flexion (f3) & $97.2 \pm 1.5$ & $89.7 \pm 0.5$ & $91.5 \pm 1.2$ & $89.6 \pm 1.4$ & $89.1 \pm 1.2$ \\
\hline Index flexion (f4) & $98.8 \pm 1.2$ & $89.5 \pm 1.3$ & $92.3 \pm 1.6$ & $89.7 \pm 0.9$ & $89.3 \pm 1.3$ \\
\hline Little extension (e1) & $97.2 \pm 1.3$ & $90.1 \pm 1.7$ & $91.7 \pm 1.2$ & $88.6 \pm 1.1$ & $89.1 \pm 0.9$ \\
\hline Ring extension (e2) & $93.6 \pm 0.8$ & $85.4 \pm 1.6$ & $89.4 \pm 1.4$ & $87.3 \pm 1.2$ & $87.1 \pm 1.4$ \\
\hline Middle extension (e3) & $92.9 \pm 1.4$ & $86.0 \pm 0.4$ & $89.0 \pm 1.3$ & $86.2 \pm 1.1$ & $86.0 \pm 1.0$ \\
\hline Index extension (e4) & $97.5 \pm 1.6$ & $90.1 \pm 1.2$ & $92.8 \pm 1.6$ & $89.9 \pm 1.4$ & $89.7 \pm 1.5$ \\
\hline Thumb flexion (f5) & $98.8 \pm 1.1$ & $92.6 \pm 1.4$ & $94.1 \pm 1.0$ & $90.5 \pm 1.2$ & $90.1 \pm 1.2$ \\
\hline Thumb extension (e5) & $95.6 \pm 1.2$ & $88.5 \pm 0.8$ & $90.5 \pm 1.1$ & $88.1 \pm 0.9$ & $87.7 \pm 1.1$ \\
\hline Thumb abduction (a5) & $94.6 \pm 1.5$ & $87.1 \pm 0.5$ & $91.2 \pm 1.2$ & $89.3 \pm 1.1$ & $88.9 \pm 1.3$ \\
\hline Average accuracy (\%) & $96.6 \pm 1.3$ & $89.16 \pm 1.1$ & $91.92 \pm 1.3$ & $89.36 \pm 1.0$ & $88.95 \pm 1.2$ \\
\hline
\end{tabular}

to find the smallest number of sEMG sensors that achieve a performance that is indistinguishable from that obtained using all available sensors. This helps us to find out which subset of sensors provides the best tradeoff between accuracy and number of sensors for each individual participant.

Both SFS and sensor elimination technique (greedy search algorithm) were implemented for 12 finger movement classes for the five amputee persons. For the sEMG subset selected by the SFS method, first 3 trials were combined in one file to produce the training set while the rest of the trials were used as testing set. For the sensor elimination technique, 11 iterations of the sensor elimination approach were performed.

TABLE III (A)

CONFUSION MATRIX (\%) FOR SUBJECT 2 (TR2) USING SENSORS 1, 3, 7, 9

\section{Predicted Movement}

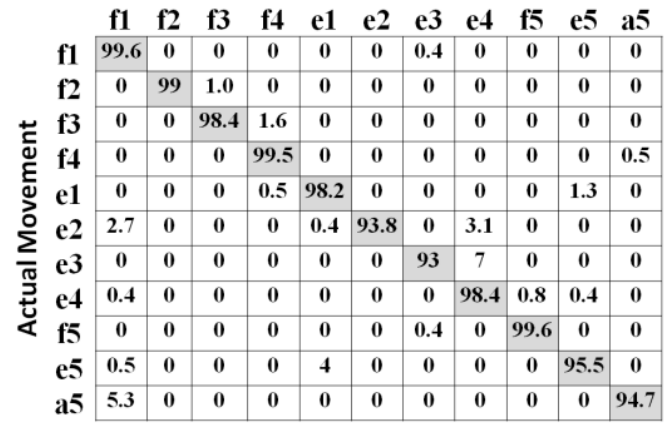

TABLE III (B)

CONFUSION MATRIX (\%) FOR SUBJECT 2 (TR2) USING SENSORS 2, 3, 7, 9 Predicted Movement

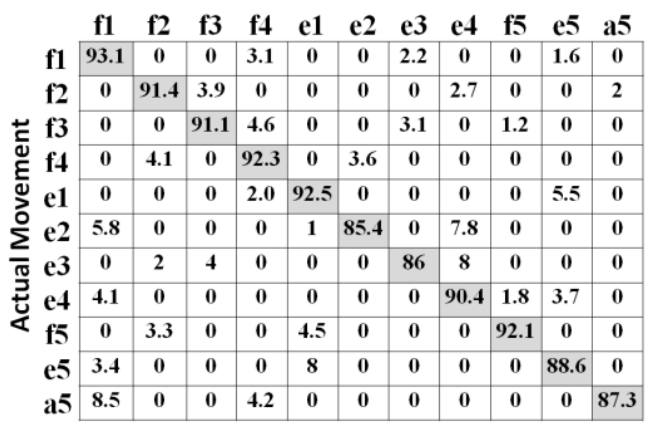

TABLE III (C)

CONFUSION MATRIX (\%) FOR SUBJECT 2 (TR2) USING ALL SENSORS - ICA

\section{Predicted Movement}

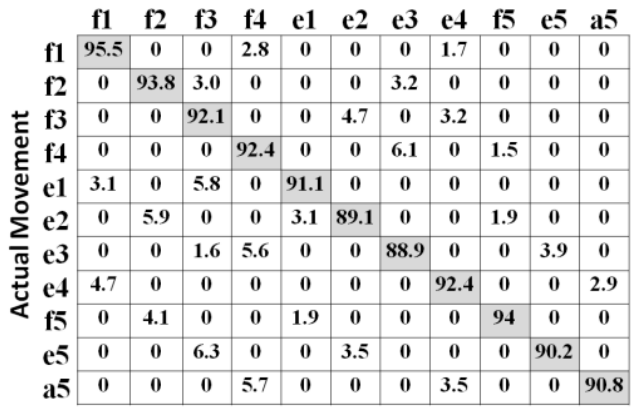

TABLE III (D)

CONFUSION MATRIX (\%) FOR SUBJECT 2 (TR2) USING SFS SCHEME Predicted Movement

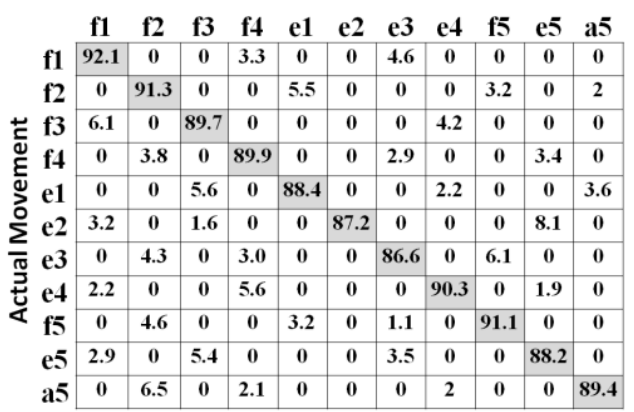

TABLE III (E)

CONFUSION MATRIX (\%) FOR SUBJECT 2 (TR2) USING GREEDY SEARCH METHOD

Predicted Movement

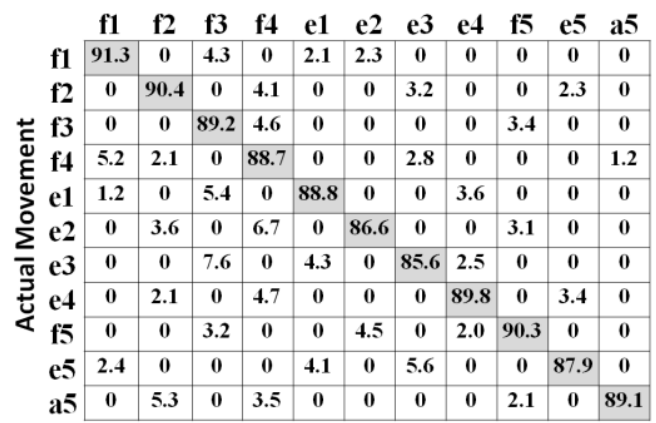


Within each iteration, the classification accuracy was calculated after eliminating one EMG sensor at a time. Then, the sensor that has the least contribution to the classification performance was removed.

\section{RESULTS AND DISCUSSION}

The overall average classification accuracies for 2 sensors combinations $(2,3,7,9$, ) and $(1,3,7,9$,), for all sensorsusing ICA, SFS and greedy search algorithm are shown in Table II. The overall classification performances of different number of sEMG sensors using Icasso clustering, SFS and greedy search algorithm are shown in Fig. 4 (a) and four sensor sensors combinations $(2,3,7,9$, and 1, 3, 7, 9) in Fig 4 (b) respectively. In addition, the classification errors for amputee subject 2 (TR2) in terms of confusion matrices for the proposed method, ICA separated data, SFS and greedy search algorithm are shown in Table III (a, b, c, d, e).

Based on Table III and IV, it can be seen that the sensor combination 1, 3, 7, 9 gives higher classification accuracy as compared to all sensors and sensor combination 2,3,7,9. This is due to the fact that sensor 1 has appeared more times than sensor 2 in the Icasso clusters. The reason for this is the centrotypes derived from clusterisation of estimate sources obtained with a reasonable numbers of trials represent the best estimates available for the real sources. Furthermore, reliable components produced a "tight" cluster of estimated components that are very close to each other and well separated from the rest. This enabled us to determine the best sensor combination for the proposed amputee gesture recognition scheme.

The outcome of this research showed an overall accuracy of $>90 \%$ for the classification of 11 finger movements using fewer sensors. It is interesting to note that, as compared to the proposed method, when only 4 sensors were used the other three methods demonstrated classification accuracy of around $75 \%$ (Refer to Fig. 4a). Moreover, both SFS and Greedy search algorithms provided reasonably good accuracy when using all the sensors (Refer to Table III) however, they failed to demonstrate good accuracy while eliminating sEMG sensors (Refer to Fig. 4a). This demonstrated the efficacy of the proposed method over the other existing techniques. In particular, the proposed system can be applied in a practical setting to make transradial amputated subjects able to control most everyday tasks in dexterous robotic hand prosthesis with no prior experience. Further training the patient and the system are expected to further improve the classification results. The results of the statistical test showed that all pvalues obtained from the controls' results were significant ( $p$ value $<0.05)$. This indicates that, there is a significant difference in the classification performance between the two sensor combination schemes $(1,3,7,9$ and 2,3,7,9), (1,3,7,9 and SFS), and (1,3,7,9 and Greedy search algorithm), respectively.

These overall results indicate that applying the proposed ICA clustering method to the sEMG dataset showed the highest classification accuracy and outperformed the other two sensor selection/elimination methods and full set of sensors.
Interestingly, the sensors used in the proposed method (Icasso selected sensors) 1,3,7,9 are clinically significant as they are closely connected to Brachioradialis, Flexor Carpi Radialis, Flexor Digitorum Superficialis and Flexor Carpi Ulnaris, which are responsible for most of the flexion and abduction tasks.

In general, using more sEMG sensors could capture more electrophysiological information that may improve the overall performance of the movement classification of sEMG based PR systems. However, this would simultaneously increase the complexity and the cost of the sEMG controlled systems. Hence, it is necessary and also important find an appropriate number of sEMG sensors and their locations for the high performance of sEMG based prosthetic devices before it is clinically viable. In this regard the proposed ICA clustering method shown promising results, however, still the algorithm needs to be tested in real-time before it is clinically applicable for the amputees.

The calibration procedure with the proposed ICA clustering technique for the EMG sensors may start by placing the full set of sensors at the amputee stump. Then, with the use of the proposed ICA clustering, the optimal reduced subset of EMG sensors will be identified. After finding the optimal EMG sensors with their locations, these sensors can be fitted permanently inside prosthesis socket by the prosthetist. The previous step is currently used in clinical practice. The amputee will wear the prosthesis for everyday use with the fitted optimized electrodes. Reducing the number of EMG sensors will minimize discomfort; reduce hardware and computational complexity as well as minimizing the cost. The above procedure based on ICA clustering can be used to fit large number of EMG sensors unlike the currently used protocol to fit only single or 2 EMG sensors [34].

\section{A. Effect of the proposed scheme on amputation time}

At present, myoelectric prostheses empower amputees to perform few dexterous movements. However, the control possibilities are usually not natural and still limited. In the recent past, there have been significant improvements over the conventional myoelectric control strategy but these results can be further improved by a general description of the problem that include the combination of innovative machine learning algorithms, pattern recognition methods and the effect of clinical parameters (level of the amputation, dominance and time interval between amputation and rehabilitation) related to the amputation [38].

The classification accuracy does not change (affect) much with the time elapsed since the amputation as shown in Fig. 5. This result shows that the subjects could control the muscles despite central reorganization that takes place after amputation, in accordance to what described for the somatosensory path $[39,40]$. In particular, we suggest that the increase in classification accuracy may be dependent on the natural reinnervation of the remnant muscles in the forearm. Moreover, results demonstrate that even subjects amputated few years ago can achieve good control of multifunctional upper limb prostheses. 

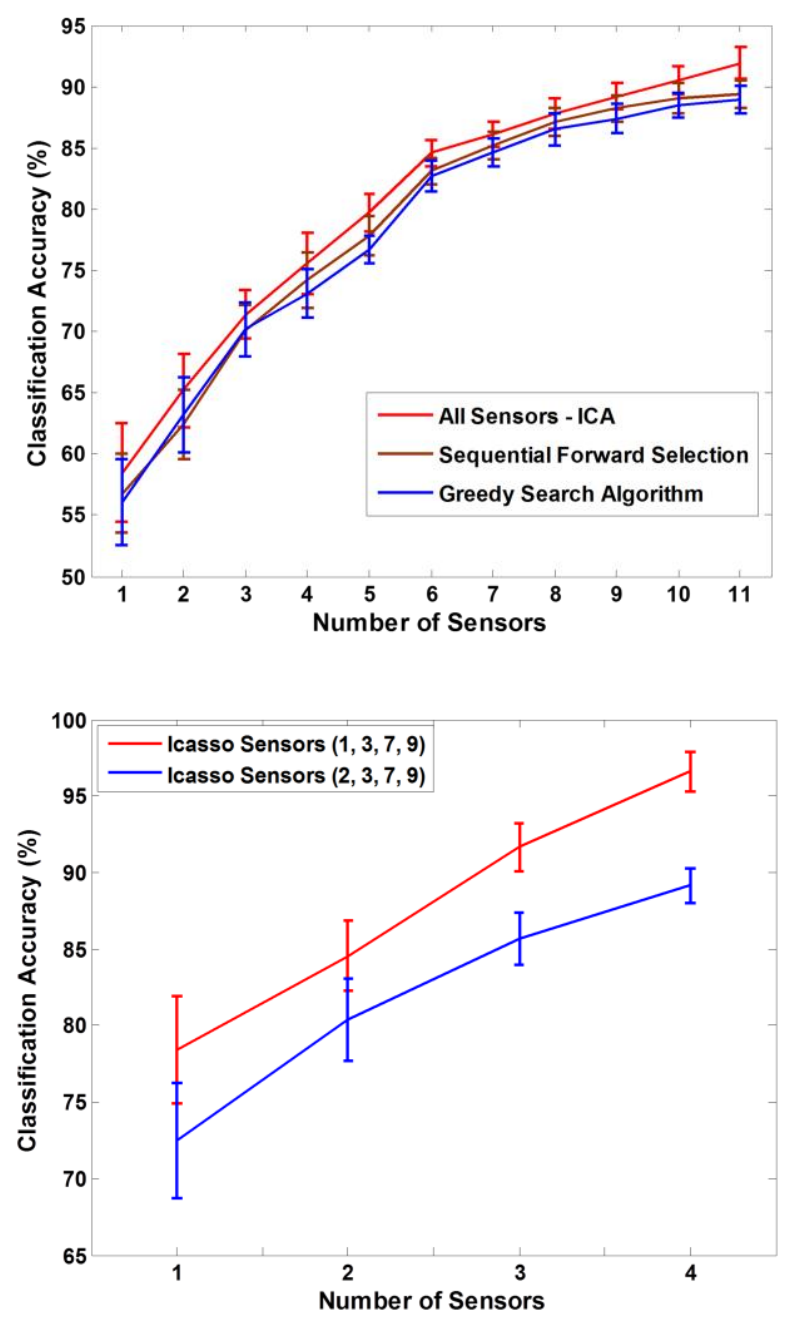

Fig. 4(a). Average classification accuracy for different number of sEMG sensors using: i) All Sensors - ICA, ii) SFS and iii) greedy search algorithm. 4(b). Average classification accuracy for 4 sEMG sensors using the proposed scheme: i) Sensors $(1,3,7,9)$ and ii) Sensors $(2,3,7,9)$

\section{B. Computational complexity measure}

The computational complexity of the proposed method was measured using the ratio of $N_{m} / N_{c h}$, where $N_{m}$ is the number of finger movements and $N_{c h}$ is the number of sEMG sensors [7].Table IV presents a summary of the $N_{m} / N_{c h}$ ratio for the proposed method and other benchmark methods presented in the paper. Here we achieved a highest ratio (2.75) for the proposed method as compared to the other methods and also with higher classification accuracy. This shows that the sensor selection method used in the study is less computationally intensive than other sensor selection methods since it has the benefit of selecting the best sensors based on source separation and Icasso clustering method.

Several pattern recognition and machine learning application exist to analyse amputee sEMG data. The proposed research has potential to minimize the number of sensors required for EMG prosthetics; however, this scheme needs to be tested with larger number of amputees' data in order to realize its full potential. The study has limitation where only offline analysis was used to test the classification performance of PR system with the use of Icasso technique.
TABLE IV

RESEARCH Illustrating THE NUMBER OF SEMG SENSORS USED, THE NuMBER OF Finger MOVEMENTS Classified, THE Classification ACCURACY AND THE RATIO OF THE NUMBER OF MOVEMENTS DECODED $\left(N_{m}\right)$ DIVIDED BY THE NUMBER OF EMG SENSORS $\left(N_{c h}\right)$

\begin{tabular}{|c|c|c|c|c|}
\hline Method & $N_{c h}$ & $N_{m}$ & $\begin{array}{c}\text { Overall Classification } \\
\text { accuracy }\end{array}$ & $N_{m} / N_{c h}$ \\
\hline SFS & 11 & 11 & $89.36 \pm 1.01$ & 1 \\
\hline GREEDY SEARCH & 11 & 11 & $88.95 \pm 1.16$ & 1 \\
\hline ICA & 11 & 11 & $91.92 \pm 1.29$ & 1 \\
\hline $\begin{array}{c}\text { PROPOSED } \\
\text { SENSORS 2,3,7,9 }\end{array}$ & 4 & 11 & $89.16 \pm 1.1$ & 2.75 \\
\hline $\begin{array}{c}\text { PROPOSED } \\
\text { (SENSORS 1,3,7,9) }\end{array}$ & 4 & 11 & $96.6 \pm 1.3$ & 2.75 \\
\hline
\end{tabular}

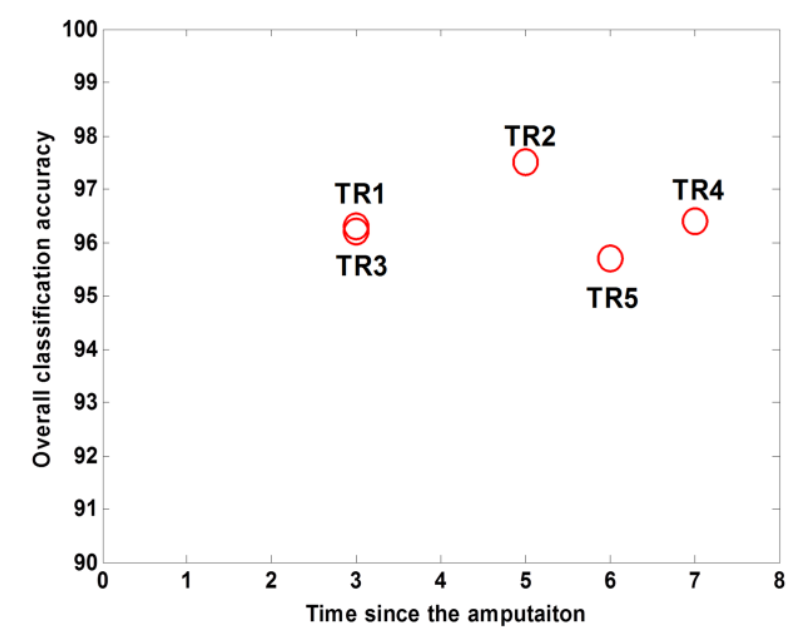

Fig. 5. Graph showing the overall classification accuracy vs. time passed since the amputation in years for five amputee subjects.

The proposed method was not evaluated in real situations such as object grasping, pinching etc. Both robustness and reliability of the proposed method needs to be validated with a systematic analysis, preferably using more number of amputees, as it was shown that off-line accuracy and online performance of pattern recognition algorithm did not necessarily correlate [41].

\section{CONCLUSION}

In this paper, we propose combination of ICA and Icasso method to minimize the number of SEMG sensors and also to improve the robustness of myoelectric control system. Advantages of using ICA and Icasso as a pre-processing step are twofold; (i) it helps in reducing the number of sensors and (ii) using this approach, only the best sensors responsible for hand and finger actions are selected.

The main contributions of this research are the following: (i) we introduced combination of source separation and model based technique to minimise the number of sEMG sensors, and (ii) classified 11 gestures for amputees with $>90 \%$ classification accuracy. Consequently, this study has the potential for improving the usability and practicality of PR system in a realistic scenario and makes it clinically viable option. Moreover, the proposed technique could be tailored with more functional prosthetic arms using current machine learning approaches and most efficient rehabilitation programs.

The amputation of the upper extremity, especially 
transradial amputation is a severely impairing injury. As described in scientific literature, sEMG is a promising method to control non-invasive, dexterous, robotic prosthetic hands but still large numbers of EMG sensors are needed to achieve good performance. In this study, we tried to tackle this problem and propose a solution with the use of Icasso in order facilitate the design of practical PR systems toward the ultimate goal clinical implementation.

Future research will be to develop a real time myoelectric system based on the proposed scheme and test the robustness of the individual features such as electrode shift, electrode size and orientation, on amputees. Furthermore, the proposed EMG sensor minimization technique will be implemented on hardware (prosthetic device) and with the help of clinicians and prosthetic experts appropriate clinical trials will be performed on amputees.

\section{ACKNOWLEDGMENT}

The authors would like to thank the amputees for their participation, Sarmad Haitham and Hussein Al-Jamal for recruiting the amputees and also Hai $\mathrm{Li}$ and Nicholas Outram for building the EMG amplifier. The authors are also grateful to Adrian Chan for providing the Myoelectric Control Toolbox.

\section{REFERENCES}

[1] J. Hahne, F. Biebmann, N. Jiang, H. Rehbaum, D. Farina, F. Meinecke, K.-R. Muller, and L. Parra, "Linear and Nonlinear Regression Techniques for Simultaneous and Proportional Myoelectric Control," IEEE Transactions on Neural Systems and Rehabilitation Engineering, vol. 22, pp. 269-279, 2014.

[2] A. J. Young, L. H. Smith, E. J. Rouse, and L. J. Hargrove, "Classification of simultaneous movements using surface EMG pattern recognition," IEEE Transactions on Biomedical Engineering, vol. 60, pp. 1250-1258, 2013.

[3] A. Fougner, O. Stavdahl, P. J. Kyberd, Y. G. Losier, and P. A. Parker, "Control of upper limb prostheses: terminology and proportional myoelectric control-a review," IEEE Transactions on Neural Systems and Rehabilitation Engineering, vol. 20, pp. 663-677, 2012.

[4] A. D. Roche, H. Rehbaum, D. Farina, and O. C. Aszmann, "Prosthetic Myoelectric Control Strategies: A Clinical Perspective," Current Surgery Reports, vol. 2, pp. 1-11, 2014.

[5] E. A. Biddiss and T. T. Chau, "Upper limb prosthesis use and abandonment: A survey of the last 25 years," Prosthetics and orthotics international, vol. 31, pp. 236-257, 2007.

[6] R. Clement, K. Bugler, and C. Oliver, "Bionic prosthetic hands: A review of present technology and future aspirations," The surgeon, vol. 9, pp. 336-340, 2011.

[7] A. H. Al-Timemy, G. Bugmann, J. Escudero, and N. Outram, "Classification of Finger Movements for the Dexterous Hand Prosthesis Control with Surface Electromyography," IEEE Journal of Biomedical and Health Informatics, vol. 17, pp. 608-618, 2013.

[8] L. Liu, P. Liu, E. A. Clancy, E. Scheme, and K. B. Englehart, "Electromyogram whitening for improved classification accuracy in upper limb prosthesis control," IEEE Transactions on Neural Systems and Rehabilitation Engineering, vol. 21, pp. 767-774, 2013.

[9] J. Liu, X. Li, G. Li, and P. Zhou, "EMG feature assessment for myoelectric pattern recognition and channel selection: A study with incomplete spinal cord injury," Medical engineering \& physics, vol. 36, pp. 975-980, 2014.

[10] E. E. Scheme, K., "Electromyogram pattern recognition for control of powered upper-limb prostheses: State of the art and challenges for clinical use," Journal of Rehabilitation Research \& Development (JRRD), vol. 48, pp. 643-660, 2011.
[11] R. N. Khushaba, M. Takruri, J. V. Miro, and S. Kodagoda, "Towards limb position invariant myoelectric pattern recognition using timedependent spectral features," Neural Networks, vol. 55, pp. 42-58, 2014.

[12] A. H. Al-Timemy, G. Bugmann, J. Escudero, and N. Outram, "A preliminary investigation of the effect of force variation for myoelectric control of hand prosthesis," in 35th Annual International Conference of the IEEE Engineering in Medicine and Biology Society (EMBC), 2013 2013, pp. 5758-5761.

[13] J. He, D. Zhang, X. Sheng, S. Li, and X. Zhu, "Invariant Surface EMG Feature Against Varying Contraction Level for Myoelectric Control Based on Muscle Coordination," IEEE Journal of Biomedical and Health Informatics, vol. 19, pp. 874-882, 2014.

[14] A. H. Al-Timemy, "An investigation of electromyographic (EMG) control of dextrous hand prostheses for transradial amputees," $\mathrm{PhD}$, School of Computing and Mathematics, Plymouth University, U.K, 2013

[15] N. Jiang, S. Dosen, K.-R. Müller, and D. Farina, "Myoelectric control of artificial limbs-Is there a need to change focus," IEEE Signal Processing Magazine, vol. 29, pp. 152-150, 2012.

[16] H. Huang, P. Zhou, G. Li, and T. A. Kuiken, "An analysis of EMG electrode configuration for targeted muscle reinnervation based neural machine interface," IEEE Transactions onNeural Systems and Rehabilitation Engineering, vol. 16, pp. 37-45, 2008.

[17] Y. Geng, X. Zhang, Y.-T. Zhang, and G. Li, "A novel channel selection method for multiple motion classification using high-density electromyography," Biomedical engineering online, vol. 13, p. 102, 2014.

[18] T. R. Farrell, "Determining delay created by multifunctional prosthesis controllers," Journal of rehabilitation research and development, vol. 48, 2011.

[19] A. J. Young, L. J. Hargrove, and T. A. Kuiken, "Improving myoelectric pattern recognition robustness to electrode shift by changing interelectrode distance and electrode configuration," IEEE Transactions on Biomedical Engineering, vol. 59, pp. 645-652, 2012.

[20] G. R. Naik and D. K. Kumar, "Estimation of independent and dependent components of non-invasive EMG using fast ICA: validation in recognising complex gestures," Computer methods in biomechanics and biomedical engineering, vol. 14, pp. 1105-1111, 2011.

[21] G. R. Naik, S. Arjunan, and D. Kumar, "Applications of ICA and fractal dimension in sEMG signal processing for subtle movement analysis: a review," Australasian Physical \& Engineering Sciences in Medicine, vol. 34, pp. 179-193, 2011.

[22] G. R. Naik and D. K. Kumar, "Identification of hand and finger movements using multi run ICA of surface electromyogram," Journal of medical systems, vol. 36, pp. 841-851, 2012.

[23] A. Hyvärinen, J. Karhunen, and E. Oja, Independent component analysis vol. 46: John Wiley \& Sons, 2004.

[24] J. Himberg, A. Hyvärinen, and F. Esposito, "Validating the independent components of neuroimaging time series via clustering and visualization," Neuroimage, vol. 22, pp. 1214-1222, 2004.

[25] E. Briselli, G. Garreffa, L. Bianchi, M. Bianciardi, E. Macaluso, M. Abbafati, M. Grazia Marciani, and B. Maraviglia, "An independent component analysis-based approach on ballistocardiogram artifact removing," Magnetic Resonance Imaging, vol. 24, pp. 393-400, 2006.

[26] G. Gómez-Herrero, M. Atienza, K. Egiazarian, and J. L. Cantero, "Measuring directional coupling between EEG sources," Neuroimage, vol. 43, pp. 497-508, 2008.

[27] A. E. Mechling, N. S. Hübner, H.-L. Lee, J. Hennig, D. von Elverfeldt, and L.-A. Harsan, "Fine-grained mapping of mouse brain functional connectivity with resting-state fMRI," Neuroimage, vol. 96, pp. 203$215,2014$.

[28] J. J. Remes, T. Starck, J. Nikkinen, E. Ollila, C. F. Beckmann, O. Tervonen, V. Kiviniemi, and O. Silven, "Effects of repeatability measures on results of fMRI sICA: a study on simulated and real resting-state effects," Neuroimage, vol. 56, pp. 554-569, 2011.

[29] G. R. Naik, K. G. Baker, and H. T. Nguyen, "Dependency Independency measure for posterior and anterior EMG sensors used in simple and complex finger flexion movements: Evaluation using SDICA," IEEE Journal of Biomedical and Health Informatics, 2014.

[30] T.-W. Lee, Independent component analysis: Springer, 1998.

[31] B. Freriks and H. Hermens, European recommendations for surface electromyography: results of the SENIAM project: Roessingh Research and Development, 2000.

[32] L. Hargrove, K. Englehart, and B. Hudgins, "A training strategy to reduce classification degradation due to electrode displacements in 
pattern recognition based myoelectric control," Biomedical Signal Processing and Control, vol. 3, pp. 175-180, 2008.

[33] A. Phinyomark, P. Phukpattaranont, and C. Limsakul, "Feature reduction and selection for EMG signal classification," Expert Systems with Applications, vol. 39, pp. 7420-7431, 2012.

[34] D. Farina, N. Jiang, H. Rehbaum, A. Holobar, B. Graimann, H. Dietl, and O. C. Aszmann, "The extraction of neural information from the surface EMG for the control of upper-limb prostheses: Emerging avenues and challenges," IEEE Transactions on Neural Systems and Rehabilitation Engineering vol. 22, pp. 797-809, 2014.

[35] R. Boostani and M. H. Moradi, "Evaluation of the forearm EMG signal features for the control of a prosthetic hand," Physiological measurement, vol. 24, p. 309, 2003.

[36] J. Ye, "Characterization of a family of algorithms for generalized discriminant analysis on undersampled problems," in Journal of Machine Learning Research, 2005, pp. 483-502.

[37] A. D. Chan and G. C. Green, "Myoelectric control development toolbox," in Proceedings of 30th Conference of the Canadian Medical \& Biological Engineering Society, 2007, pp. M0100-1.

[38] R. Casale, L. Alaa, M. Mallick, and H. Ring, "Phantom limb related phenomena and their rehabilitation after lower limb amputation," European journal of physical and rehabilitation medicine, vol. 45, pp. 559-566, 2009.

[39] P. Schwenkreis, K. Witscher, F. Janssen, B. Pleger, R. Dertwinkel, M. Zenz, J.-P. Malin, and M. Tegenthoff, "Assessment of reorganization in the sensorimotor cortex after upper limb amputation," Clinical neurophysiology, vol. 112, pp. 627-635, 2001.

[40] R. M. Libbin and M. E. Rivera, "responsiveness of the somatosensory system after nerve injury and amputation in the human hand," Annals of Neurology, vol. 36, pp. 68-75, 2004.

[41] B. Lock, K. Englehart, and B. Hudgins, "Real-time myoelectric control in a virtual environment to relate usability vs. accuracy," Fredericton, New Brunswick, Canada, 2005, pp. 1-4.

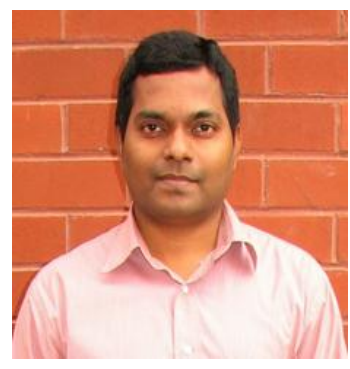

Ganesh R. Naik (M'08, SM'15) received a B.E. degree in Electronics and Communication Engineering from the University of Mysore, India, in 1997, an M.E. degree in Communication and Information Engineering from Griffith University, Brisbane, Australia, in 2002, and a $\mathrm{PhD}$ degree in Electronics Engineering, specialising in biomedical engineering and signal processing from RMIT University, Melbourne, Australia, in 2009.

Since 2013, he is working as a Chancellor's Postdoctoral Research Fellow in the Faculty of Engineering and Information Technology (FEIT), UTS. As an early career researcher, he has edited 10 books, authored more than 80 papers in peer reviewed journals, conferences, and book chapters over the last seven years. Dr. Naik serves as an associate editor for IEEE ACCESS and two Springer journals (Circuits, Systems, and Signal Processing and Australasian Physical \& Engineering Sciences in Medicine). He is a recipient of the Baden-Württemberg Scholarship from the University of Berufsakademie, Stuttgart, Germany (20062007). In 2010, Dr. Naik was awarded an ISSI overseas fellowship from Skilled Institute Victoria, Australia.

Ali H. Al-Timemy (S'11, M '14) received the B.Sc. and M.Sc degrees in medical engineering from Nahrain University, Baghdad, Iraq, in 2003 and 2006, respectively. He obtained a $\mathrm{PhD}$ degree from the Centre for Robotics and Neural Systems
(CRNS), Plymouth University, U.K. in 2013. He is a Lecturer at the Biomedical Engineering Department, Al-Khwarzmi College of Engineering/ University of Baghdad, Iraq. He is also a visiting research fellow at the CRNS, Plymouth University. His research interests include biomedical signal processing and myoelectric control of prostheses.

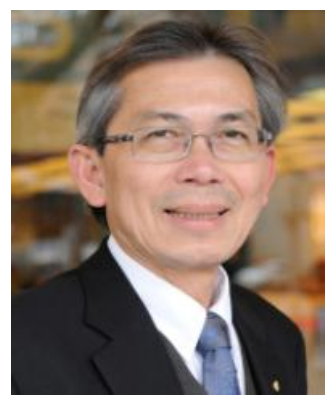

Hung T. Nguyen (SM'99) received the B.E. degree (with First Class and University Medal) and the Ph.D. degree from the University of Newcastle, Newcastle, Australia, in 1976 and 1980, respectively.

$\mathrm{He}$ is a Professor of electrical engineering at the University of Technology Sydney (UTS), Ultimo, Australia. $\mathrm{He}$ is Assistant Deputy Vice Chancellor (Innovation) and Director of the Centre for Health Technologies at the UTS. He has been involved with research in the areas of biomedical engineering, advanced control and artificial intelligence for more than 20 years. He has developed several biomedical devices and system for diabetes, disability, cardiovascular diseases, and breast cancer. He was an Engineering Manager of Power Electronics Pty Ltd., from 1988 to 1998, the Founding and Executive Director of AIMedics Pty Ltd., from 2001 to 2006, and Dean of the Faculty of Engineering and Information Technology, from 2009 to 2014.

Prof. Nguyen is a Fellow of the Institution of Engineers, Australia and the British Computer Society. 